\title{
Methods of analysis of geomagnetic field variations and cosmic ray data
}

\author{
Oksana V Mandrikova ${ }^{1,2,3^{*}}$, Igor S Solovev ${ }^{1,2,3}$ and Timur L Zalyaev ${ }^{1}$
}

\begin{abstract}
In the present paper, we propose a wavelet-based method of describing variations in the Earth's magnetic field, such as the horizontal component of the geomagnetic field, in addition to methods for evaluating changes in the energy characteristics of the field and for isolating the periods of increased geomagnetic activity. Based on a combination of multiresolution wavelet decompositions with neural networks, we propose a method of approximation of the cosmic ray time course and the allocation of anomalous variations (Forbush effects) that occur during periods of high solar activity. During the realization of the method, an algorithm was created for selecting the level of the wavelet decomposition and adaptive construction of the neural network. By using the proposed methods, we performed a joint analysis of the geomagnetic field and cosmic rays during periods of strong magnetic storms. The strongest geomagnetic field perturbations were observed in periods of abnormal changes in cosmic ray level. Assessment of the intensity of geomagnetic disturbances on the eve of and during magnetic storm development allowed us to highlight local increases in intensity of the geomagnetic field occurring at different frequency ranges prior to the development of the storm's main phase. Implementation of the proposed method with theoretical tools in combination with other methods will improve the estimation accuracy of the geomagnetic field state during space weather forecasting.
\end{abstract}

Keywords: Geomagnetic field; Cosmic rays; Wavelet transform; Neural networks; Magnetic storms

\section{Findings} Introduction

The present study examines the processes that occur in circumterrestrial space during magnetic perturbations by creating new algorithms and techniques for recognition, analysis, and interpretation of data. During perturbed periods, parameters determined by special equipment include a complex non-stationary structure that contains non-smooth local features arising at random time points that carry information about the processes under study. A lack of theoretical basis for providing adequate presentation of data for analysis leads to inevitable loss and distortion of information and requires the application of contemporary techniques such as pattern recognition and digital signal processing (Nayar et al. 2006; Hafez et al.

\footnotetext{
* Correspondence: oksanam1@mail.ru

${ }^{1}$ Institute of Cosmophysical Researches and Radio Wave Propagation,

Mirnaya Str., 7, Kamchatka Region, Elizovskiy District, Paratunka 684034, Russia

${ }^{2}$ Kamchatka State Technical University, Klyuchevskaya Street 35, 683003

Petropavlovsk-Kamchatsky, Russia

Full list of author information is available at the end of the article
}

2013; Xu et al. 2008; Jach et al. 2006; Paschalis et al. 2013; Macpherson et al. 2001; Woolley et al. 2010; Soloviev et al. 2012; Rotanova et al. 2004).

During periods of magnetic storms in the recorded geomagnetic data, variations occur among frequency ranges. The emerging local structures are determined by field perturbations and reveal the intensity and nature of the development of a magnetic storm. The complex structure of geomagnetic data limits the abilities of spectral analysis methods by failing to provide information on the local changes occurring in the physical process and their scale characteristics.

The disadvantages of these methods also include limitations in identifying and interpreting hidden patterns in the behavior of the data. In the present study, wavelet transform is suggested for analysis of geomagnetic data because such methods allow detection of several interesting deterministic and more stochastic features in the time series of solar activity indicators (Lundstedt et al. 2005). Wavelet transform is the underlying mathematical tool used for analyzing local features occurring in the

\section{实}


geomagnetic field during intense solar flares (Ivanov et al. 2001; Rotanova et al. 2004; Nayar et al. 2006) and for developing algorithms for automatic determination of the onset of a magnetic storm (Hafez et al. 2013). Furthermore, the wavelet transform is a powerful tool for eliminating noise and excluding the periodic component caused by the Earth's rotation (Xu et al. 2008; Jach et al. 2006). A method used for allocation of anomalies in geomagnetic data was developed on the basis of the wavelet transform (Zaourar et al. 2013). The authors of the present paper created technology for automatic extraction of the unperturbed level of the horizontal component of the geomagnetic field based for the first time on wavelet transform (Mandrikova et al. 2013). This method has significantly reduced error in automatic calculation of the K-index in comparison to the adaptative smoothing method (KASm) recommended by the International Real-Time Magnetic Observatory Network (Intermagnet) and currently used by Paratunka Observatory, Kamchatka Region, Institute of Cosmophysical Researches and Radiowave Propagation, Far Eastern Branch of the Russian Academy of Sciences (IKIR FEB RAS), and Yakutsk Observatory, Yakutsk, Institute of Cosmophysical Research and Aeronomy, Siberian Branch, Russian Academy of Sciences (IKFIA SB RAS). In the present paper, the authors suggest a new technique for determining variation in the geomagnetic field by using the $\mathrm{H}$-component and present related methods of extracting geomagnetic perturbations, estimating their intensity, and determining periods of increased geomagnetic activity. The suggested theoretical tools can be implemented in the automatic mode, which is close to the real-time mode and can be adapted to various magnetic observatories.

The topology of the geomagnetic field during magnetic storms is characterized by variability of cosmic rays on the Earth's surface (Kozlov and Markov 2007), which present an integral result of various solar, heliospheric, and atmospheric phenomena with a complex structure. The most substantial changes in cosmic ray parameter are caused by emissions of the corona substance and subsequent changes in parameters of the interplanetary field and solar wind (Vecchio et al. 2012). The current study of the dynamics of cosmic ray flux includes methods of adaptive approximation, wavelet transform, and neural networks. Using neural networks for the primary processing of neutron monitor data have led to greater efficiency in the noise reduction procedure compared with median methods (Paschalis et al. 2013). Neural networks also can be used to detect abnormal features in complex functions. For example, the method of automatic detection of sudden commencement is based on neural networks (Segarra and Curto 2013). Based on a combination of wavelet transform and empirical mode decomposition, the dominant temporal scales of long-term temporal variation of cosmic rays were highlighted for periods of 11,22 , and 6 years and biennial oscillation, and their physical nature was determined (Vecchio et al. 2012). In the present study, the authors combine multiscale wavelet transforms and neural networks to develop a technique for approximating the time variation of cosmic rays and discovering anomalous changes connected with increased solar activity.

This method enables noise suppression to distinguish characteristic variations and to examine the details of their structures.

On the basis of the proposed methods, we have performed joint analysis of the geomagnetic field and cosmic rays during periods of strong magnetic storms on 5 to 7 April 2010 and 5 September 2012. During the analysis, we used 1-min geomagnetic field data from Intermagnet (www.intermagnet.org) and 1-min neutron monitor data obtained within the framework of the Realtime Neutron Monitor Database (NMDB) (www.nmdb.eu/) project.

\section{Description of methods}

Decomposition of time series into components based on wavelet transform

Having the discrete function value $f_{j}$ (i.e., values of the function at the grid with a resolution of $2^{-j}$ ), we can consider the following subspace as the sampling space:

$$
V_{j}=\operatorname{clos}_{L^{2}(R)}\left(\phi\left(2^{j} t-n\right)\right), n \in Z,
$$

where $V_{j}$ is generated by translation and dilation of the scaling function $\phi$ (Daubechies 1992; Chui 1992; Mallat $1999)$, and $L^{2}(R)$ is the Lebesgue space.

By performing the projection of $f_{j}$ into spaces $V_{j-1}$ and $W_{j-1}$, we obtain (Daubechies 1992; Chui 1992; Mallat 1999)

$$
V_{j}=V_{j-1} \oplus W_{j-1}
$$

where $\oplus$ denotes the orthogonal sum. The set of functions (wavelets) $\Psi_{j, n}=2^{j / 2} \Psi\left(2^{j} t-n\right), n \in Z$ form the basis of the space $W_{j}$.

We consider $f_{j}$ as time series. On the basis of Equation 2, the time series can be represented as:

$$
f_{j}(t)=f_{j-1}(t)+g_{j-1}(t) .
$$

Each component of the series $f_{j-1}(t)=\sum_{n} c_{n}^{j-1} \Psi_{j-1, n}(t)$ and $g_{j-1}(t)=\sum_{n} d_{n}^{j-1} \Psi_{j-1, n}(t)$ in Equation 3 is uniquely 


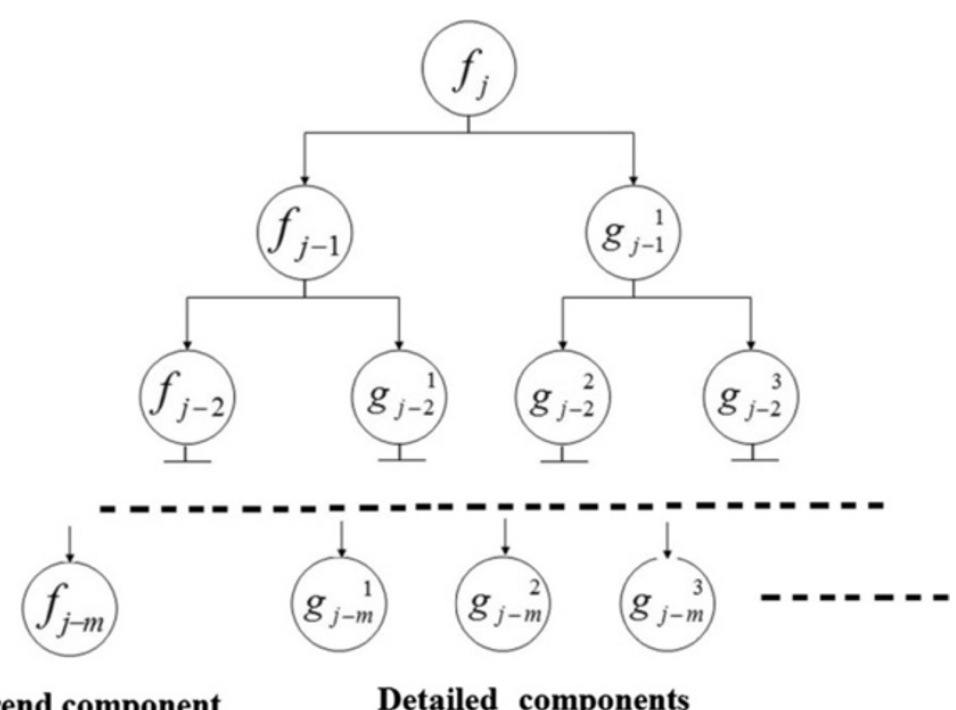

Trend component

Detailed components

Figure 1 Graphic representation of wavelet packet decomposition to the level $m$ of the time series $f_{j}$.

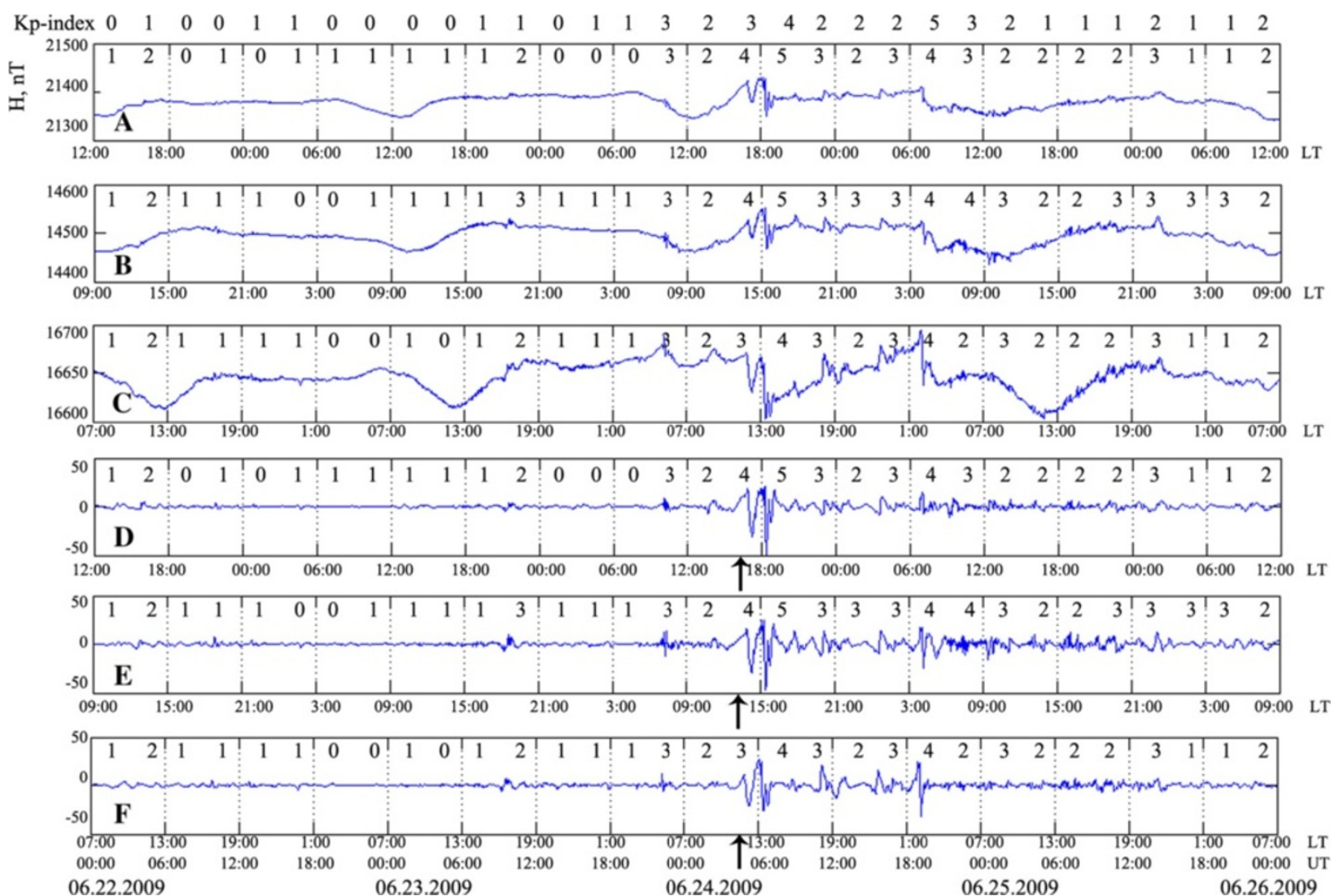

Figure 2 Results of processing of the data of geomagnetic field for the period 22 to 25 June 2009. (A) H-component of the geomagnetic field of the Earth (Paratunka), (B) H-component of the geomagnetic field of the Earth (Yakutsk), (C) H-component of the geomagnetic field of the Earth (Novosibirsk), (D) disturbed component of the geomagnetic field variations (Paratunka), (E) disturbed component of the geomagnetic field variations (Yakutsk), and (F) disturbed component of the geomagnetic field variations (Novosibirsk). Local time is marked on the horizontal axis. The top graph shows values of K-indices. 


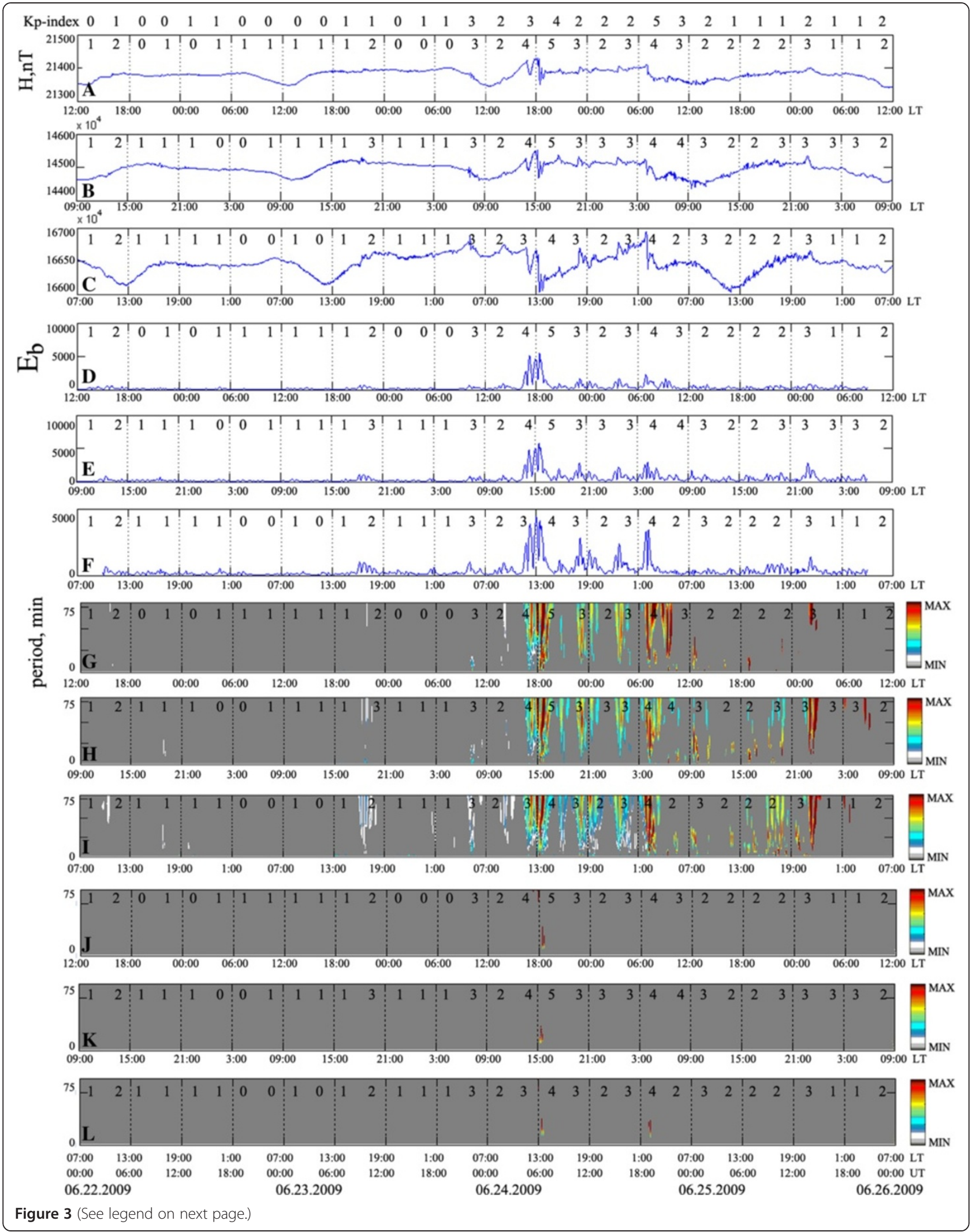


(See figure on previous page.)

Figure 3 Results of processing of the data of geomagnetic field for the period 22 to 25 June 2009. (A) H-component of the geomagnetic field of the Earth (Paratunka), (B) H-component of the geomagnetic field of the Earth (Yakustk), (C) H-component of the geomagnetic field of the Earth (Novosibirsk), (D) assessment of the intensity of geomagnetic disturbances (Paratunka), (E) assessment of the intensity of geomagnetic disturbances (Yakutsk), (F) assessment of the intensity of geomagnetic disturbances (Novosibirsk), (G) allocation of the periods of strong and weak perturbations (Paratunka), (H) allocation of the periods of strong and weak perturbations (Yakutsk), (I) allocation of the periods of strong and weak perturbations (Novosibirsk), (J) allocation of the periods of strong perturbations (Paratunka), (K) allocation of the periods of strong perturbations (Yakutsk), and (L) allocation of the periods of strong perturbations (Novosibirsk). Local time is marked on the horizontal axis. At the top of the graphs are the values of K-indices.

defined by the sequence of coefficients $\bar{c}^{j}=\left\{c_{j, n}\right\}_{n \in Z} \in V_{j}$ and $\bar{d}^{j}=\left\{d_{j, n}\right\}_{n \in Z} \in W_{j}: c_{j, n}=\left\langle f, \phi_{j, n}\right\rangle, d_{j, n}=\left\langle f, \Psi_{j, n}\right\rangle$.

The described computational procedure is known as a wavelet decomposition algorithm, which can be applied to any resolution (scale) $j$ of the signal (Daubechies 1992; Chui 1992; Mallat 1999).

If this decomposition procedure is performed on the time series $f_{j}(t) m$ times, we obtain the following form of the decomposition of the space $V_{j}$ :

$$
V_{j}=W_{j-1} \oplus W_{j-2} \oplus \ldots \oplus W_{j-m} \oplus V_{j-m} .
$$

As a result, the time series $f_{j}(t)$ can be represented as the sum of the components listed below:

$$
\begin{aligned}
f_{j}(t) & =g_{j-1}(t)+g_{j-2}(t)+\ldots+g_{j-m}(t)+f_{j-m}(t) \\
& =\sum_{k=j-1}^{j-m} \sum_{n} d_{k, n} \Psi_{k, n}(t)+\sum_{n} c_{j-m, n} \phi_{j-m, n}(t) .
\end{aligned}
$$

Component $g_{j}$ is known as a detailed (high-frequency) component of the time series; component $f_{j-m}$ is known as the smoothed component of the time series.

In the wavelet transform theory, decomposition of the form in Equation 5 is known as multiresolution wavelet decomposition to the level $m$ (Chui 1992; Mallat 1999).

If we apply this space decomposition procedure to the space $W_{j}$, we obtain a partition of the high-frequency components, which allows us to identify different types of time-frequency structures. In the wavelet transform theory, decomposition of $f_{j}$ based on recursive splitting of the spaces $V_{j}$ and $W_{j}$ is known as the wavelet packet decomposition. Graphic representation of wavelet packet decomposition of the time series $f_{j}$ is shown in Figure 1.

\section{Description of geomagnetic field variations on the basis of wavelets}

As $f_{j}(t)$, we could consider the time variation of the horizontal component of the geomagnetic field. Without loss of generality, we assume that $j=0$, i.e., the space of initial data is $V_{0}=\operatorname{clos}_{L^{2}(R)}\left(\phi\left(2^{0} t-n\right)\right)$. Based on the wavelet packet decomposition, we obtain the following representation of $f_{0}(t)$ :

$$
\begin{aligned}
f_{0}(t)= & \sum_{n} c_{-m, n} \phi_{-m, n}(t)+\sum_{j \in I} \sum_{n} d_{j, n} \Psi_{j, n}(t) \\
& +\sum_{j \neq I} \sum_{n} d_{j, n} \Psi_{j, n}(t)=f_{\text {trend }}(t)+f_{\text {dist }}(t)+e(t),
\end{aligned}
$$

where $I$ is a set of indices.

In our previous research (Mandrikova et al. 2013; Mandrikova and Solovev 2013), we showed, that, for Kamchatka and Yakutsk regions, component $f_{\text {trend }}(t)=\sum_{n} c_{-6, n} \phi_{-6, n}(t)$ describes the undisturbed level of the horizontal component of the magnetic field of the Earth, and component $f_{\text {dist }}(t)=\sum_{j \in I} g_{j}(t)$, where $g_{j}(t)=\sum_{n} d_{j, n} \Psi_{j, n}(t)$, describes perturbations, arising in periods of increasing geomagnetic activity. The component $e(t)=\sum_{j \neq I} \sum_{n} d_{j, n} \Psi_{j, n}(t)$ is the noise. We introduced variable $A_{j}=\max _{n}\left(\left|d_{j, n}\right|\right)$ as the measure of magnetic disturbance of the component $g_{j}(t)$ on the scale $j$ (Mandrikova et al. 2013; Mandrikova and Solovev 2013). We used the following criteria to determine the set of indices I (Mandrikova et al. 2013):

$$
j \in I, \text { if } m\left(A_{j}^{v}\right)>m\left(A_{j}^{k}\right)+\varepsilon,
$$

where $m$ is the sample mean, $v$ is the index of disturbed field variation, $k$ is the index of calm field variation, and $\varepsilon$ is a positive number.

Assuming that variable $A_{j}^{k}$ is normally distributed with some mean $\mu^{k}$ and variance $\sigma^{2, k}$, it is possible to estimate $\varepsilon$ as $\hat{\varepsilon}=x_{\frac{1-\alpha}{2}} \sqrt{\sigma^{2, k}}$, where $x_{\frac{1-\alpha}{2}}$ is the $\frac{1-\alpha}{2}$ quantile of standard normal distribution.

Estimation of the set of indices $I$, particularly for the station Paratunka (Kamchatka Region), was performed using geomagnetic data for 2002, 2005, and 2008, containing 63 disturbed field variations and 64 calm field variations. 


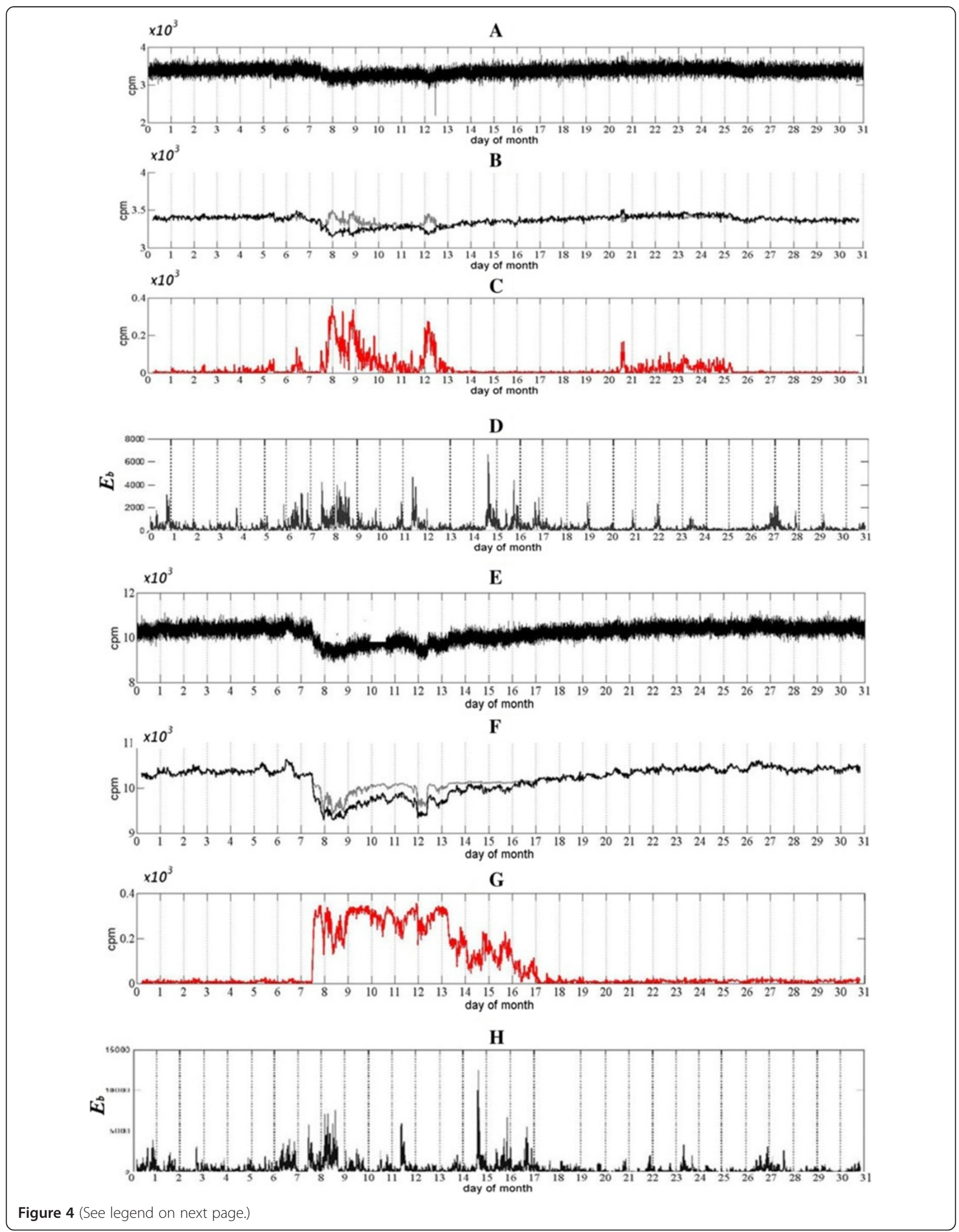


(See figure on previous page.)

Figure 4 Results of modeling of the data of cosmic rays for the period 1 to 31 March 2012. (Athens station): (A) cosmic ray time series, (B) smoothed component of cosmic ray time series (black) and its approximation by neuron network, (C) neuron network error, and (D) assessment of the intensity of geomagnetic disturbances for the geomagnetic data from Nea-Makri station (Greece). Novosibirsk station: (E) cosmic ray time series, (F) smoothed component of cosmic ray time series (black) and its approximation by neuron network, (G) neuron network error, and $\mathbf{( H )}$ assessment of geomagnetic disturbance intensity for the geomagnetic data from Novosibirsk station (Russia).

Figure 2 shows geomagnetic data processing results from stations Paratunka (Kamchatka Region), Yakutsk (Yakutsk), and Novosibirsk (Novosibirsk) for the duration of a magnetic storm that occurred 24 to 25 June 2009. The disturbed component of the geomagnetic field, allocated on the basis of the proposed model, describes the power of disturbance of the field and allows us to register the moments of increasing geomagnetic activity on the eve of and during the storms. Analysis of Figure 2 showed an increase in geomagnetic activity from 4:18 to 4:54 UT at all analyzed stations, indicating the beginning of a magnetic storm. The moments of enhancements in geomagnetic activity in Figure 2D,E,F are indicated with arrows. The maximum values of the amplitude of the disturbed component were recorded from approximately 06:08 to 06:27 UT.

Let us consider three possible states of the geomagnetic field: state $h_{0}$ : calm field; state $h_{1}$ : weakly perturbed field; state $h_{2}$ : perturbed field. According to the proposed field states, we introduce the following states of the coefficients $d_{j, n}$ on the scale $j$ :

$$
\begin{aligned}
& h_{j}^{0}: \text { calm coefficient } \\
& h_{j}^{1}: \text { weakly perturbed coefficient } \\
& h_{j}^{2}: \text { perturbed coefficient. }
\end{aligned}
$$

As a measure of magnetic disturbance of the coefficient, it is logical to determine its amplitude. Then, in accordance with the introduced states of the coefficients from Equation 6, we obtain the following representation of geomagnetic field variations:

$$
\begin{aligned}
f(t)= & f_{\text {trend }}(t)+\sum_{j, n} F_{0}\left(d_{j, n}\right) \Psi_{j, n}(t)+\sum_{j, n} F_{1}\left(d_{j, n}\right) \Psi_{j, n}(t) \\
& +\sum_{j, n} F_{2}\left(d_{j, n}\right) \Psi_{j, n}(t)+e(t), \\
F_{0}(x)= & \left\{\begin{array}{c}
x, \text { if }|x| \leq T_{j, 1} \\
0, \text { if }|x|>T_{j, 1}
\end{array}, F_{1}(x)=\left\{\begin{array}{c}
0, \text { if }|x| \leq T_{j, 1} \text { if }|x|>T_{j, 2} \\
x, \text { if } T_{j, 1}<|x| \leq T_{j, 2}
\end{array}, F_{2}(x)\right.\right. \\
= & \left\{\begin{array}{c}
0, \text { if }|x| \leq T_{j, 2} \\
x, \text { if }|x|>T_{j, 2}
\end{array}\right.
\end{aligned}
$$

where component $g_{1}(t)=\sum_{j, n} F_{1}\left(d_{j, n}\right) \Psi_{j, n}(t)$ describes weak geomagnetic perturbations, where the coefficients of $g_{1}(t)$ are in a weakly perturbed state; component $g_{2}(t)=\sum_{j, n} F_{2}\left(d_{j, n}\right) \Psi_{j, n}(t)$ describes strong geomagnetic perturbations, where the coefficients of $g_{2}(t)$ and $T_{j, 1}>T_{j, 2}$ are in a perturbed state; and coefficients $d_{j, n}$, where $\left|d_{j, n}\right| \leq$ $T_{j, 1}$ is considered as quiet.

Threshold functions $F_{0}(x), F_{1}(x)$, and $F_{2}(x)$ in Equation 9 determine rules for decision-making of coefficient state.

Threshold $T_{j, 1}$ and $T_{j, 2}$ divide the space of coefficients $X$ into three disjoint regions $X_{0}, X_{1}$, and $X_{2}$. Then, the rule of decision-making establishes a correspondence between decisions of the state of coefficients and regions.

Due to the random nature of the investigated object, the use of any rule was inevitably linked to the possibility of wrong decisions. By using certain rules for selecting a decision for a given state $h_{j}^{i}$, the average value of the loss can be defined as (Levin 1963)

$$
J_{j}^{i}(x)=\sum_{l=0}^{2} \Pi_{i l} P\left\{x \in X_{l} / h_{j}^{i}\right\}
$$

where $\Pi_{i l}$ if loss function, $P\left\{x \in X_{l} / h_{j_{\text {dist }}, i}\right\}$ is the conditional probability that the sample falls into the area $X_{l}$ if the real state is $h_{j}^{i}, i \neq l i$. $l$ is the state of indices, where sign ' $/$ ' means conditional probability.

By averaging the conditional risk function over all states $h_{j}^{i}, i=0,1,2$, we obtain average risk (Levin 1963):

$$
J=\sum_{i=0}^{2} p_{i} J_{j}^{i}
$$

where $p_{i}$ is the a priori probability of the state $h_{j}^{i}$.

The best rule is the one for which the average risk is smallest (Levin 1963).

Since we did not know the a priori distribution of states $p_{i}$, we used a posteriori risk for choosing the best decision rule (Levin 1963). Posterior probabilities $P\left\{h_{j}^{i} / x\right\}, i=0,1,2$, represent the most complete characterization of the states $h_{j}^{i}$ with available priori data.

For simple loss function:

$$
\Pi_{i l}=\left\{\begin{array}{l}
1, i \neq l, \\
0, i=l,
\end{array}\right.
$$

A posteriori risk $J_{l}(x)$ is 


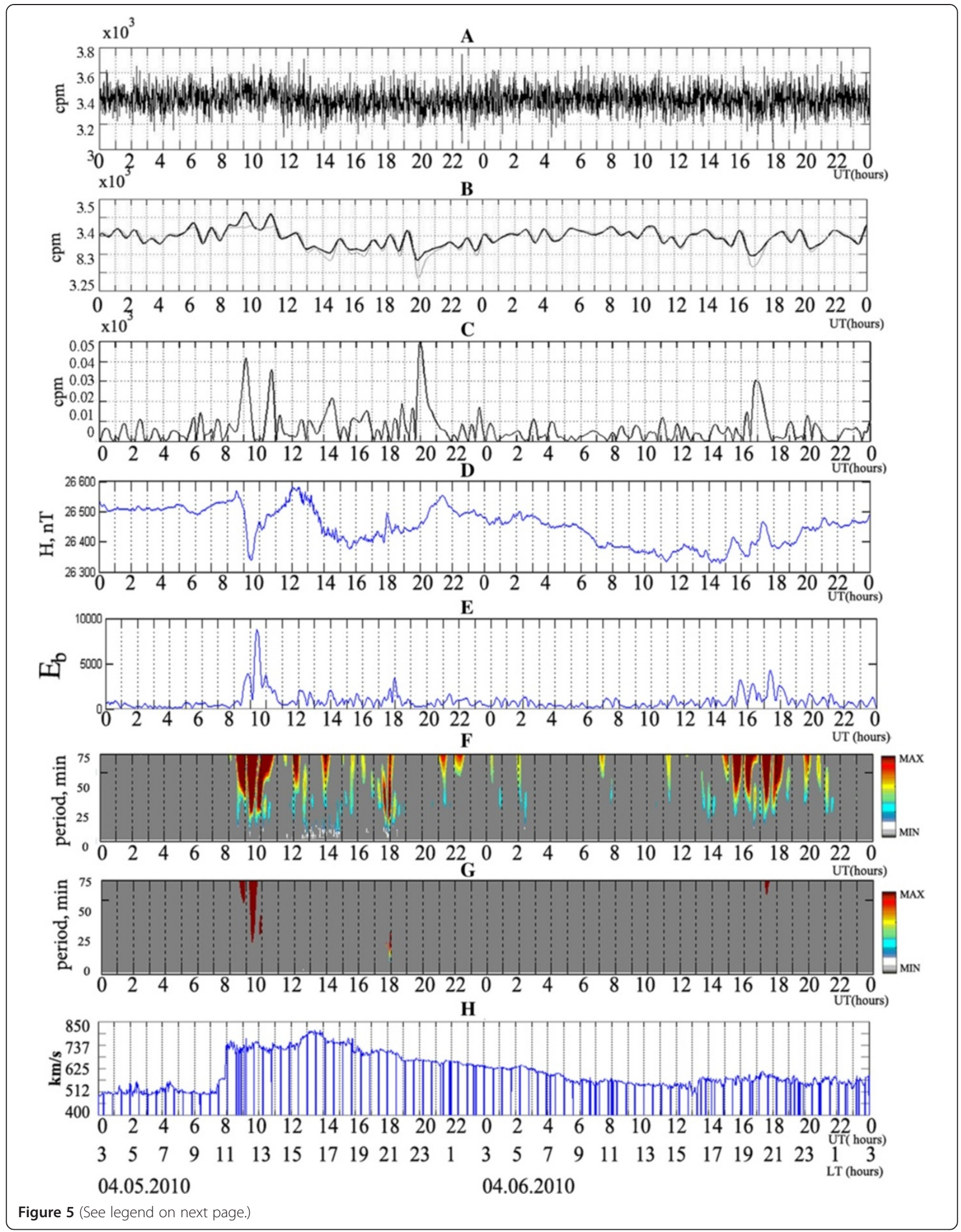


(See figure on previous page.)

Figure $\mathbf{5}$ Results of processing of the data of geomagnetic field and cosmic rays for the period $\mathbf{5}$ to $\mathbf{7}$ April 2010. Athens station:

(A) cosmic ray time series, (B) smoothed component of cosmic ray time series (black) and its approximation by neuron network, (C) neuron network error, (D) H-component of the magnetic field of the Earth at Nea-Makri station (Greece), (E) assessment of geomagnetic disturbance intensity, (F) allocation of the periods of weak and strong geomagnetic disturbances, (G) allocation of the periods of strong geomagnetic disturbances, and $(\mathbf{H})$ solar wind speed.

$$
J_{l}(x)=\sum_{i \neq l} P\left\{h_{j}^{i} / x \in X_{l}\right\}
$$

In this case, the criterion of the quality of decisionmaking is the criterion of the lowest error rate.

Thresholds $T_{j, 1}$ and $T_{j, 2}$ are determined by best decision rule of the decision-making, ensuring the lowest value of the posterior risk $J_{l}(x)$.

By minimizing the posteriori risk $J_{l}(x)$, we determine thresholds $T_{j, 1}$ and $T_{j, 2}, j \in I$ for regions of Kamchatka and Yakutsk (Mandrikova et al. 2013; Mandrikova and Solovev 2013). The criterion of assessment of the coefficients is the K-index (Bartels et al. 1939). The following assessments were considered:

1) Coefficient belongs to area $X_{0}$ (i.e., they are in a quiescent state) if the current value of the K-index is 0 or 1 ;

2) Coefficient belongs to area $X_{1}$ (i.e., they are in a weakly perturbed state) if the current value of the K-index is 2, 3, or 4;

3) Coefficient belongs to area $X_{2}$ (i.e., they are in a perturbed state) if the current value of the K-index is more than 4 .

The introduced states of coefficients describe the states of the geomagnetic field and their assessment based on the obtained rule for decision-making, which allows us to register moments of increasing geomagnetic activity. In our previous paper (Mandrikova et al. 2013), we developed an automatic algorithm to construct the Sq-curve and to calculate the K-index on the basis of this representation of geomagnetic field variations (shown in Equation 8), and we demonstrated that its application for stations Paratunka and Yakutsk significantly reduced the error of K-index computation in the automatic mode, in comparison with the methodology used in the Intermagnet network stations. In the present work ('Assessment of changes of energy characteristics of the field and the allocation of periods of increased geomagnetic activity' subsection), we used this approach to suggest computational solutions for estimating changes in the energy characteristics of the field and the allocation of periods of increased geomagnetic activity. To obtain more detailed information on the state of the geomagnetic field, we used continuous wavelet transform.

\section{Assessment of changes of energy characteristics of the field and the allocation of periods of increased geomagnetic activity}

For each of the basic wavelet, the continuous wavelet transform is defined by (Daubechies 1992; Chui 1992)

$$
\left(W_{\Psi f}\right)(b, a):=|a|^{-1 / 2} \int_{-\infty}^{\infty} f(t) \Psi\left(\frac{t-b}{a}\right) d t, f \in L^{2}(R), a, b \in R, a \neq 0 .
$$

Since wavelet $\Psi$ has a zero mean, coefficients $\left(W_{\Psi} f\right)$ $(b, a)$ describe the properties of the function $f$ in the neighborhood $b$ when the scale $a$ tends to be zero. This property of the continuous wavelet transform allows us to obtain detailed information on the local properties of the function $f$.

Using equivalence of discrete and continuous wavelet transforms (Mallat 1999; Chui 1992), it is possible to introduce a method for calculating the intensity of geomagnetic perturbations at the time point $t=b$ on the scale $a$. This intensity can be written as:

$$
e_{b, a}=\left|\left(W_{\Psi} f\right)(b, a)\right|
$$

Then, by applying a threshold function to the value $e_{b, a}$, we can estimate the state of the coefficients and allocate time-frequency intervals containing weak and strong geomagnetic disturbances on the analyzed scale $a$ :

$$
\begin{aligned}
P_{T_{a, 1}}\left(e_{b, a}\right) & =\left\{\begin{array}{l}
0, \text { if } e_{b, a}<T_{a, 1} \\
e_{b, a}, \text { if } e_{b, a} \geq T_{a, 1}
\end{array}, P_{T_{a, 2}}\left(e_{b, a}\right)\right. \\
& =\left\{\begin{array}{l}
0, \text { if } e_{b, a}<T_{a, 2}, \\
e_{b, a}, \text { if } e_{b, a} \geq T_{a, 2}
\end{array}\right.
\end{aligned}
$$

where the threshold $T_{a, 1}$ allows to us allocate weak and strong perturbations, and the threshold $T_{a, 2}$ indicates strong perturbations.

On the basis of (Equation 15), the intensity of field disturbances at the time moment $t=b$ is

$$
E_{b}=\sum_{a} e_{b, a}
$$

Figure 3 shows the results of assessment of the intensity of geomagnetic disturbances and allocation of periods of 


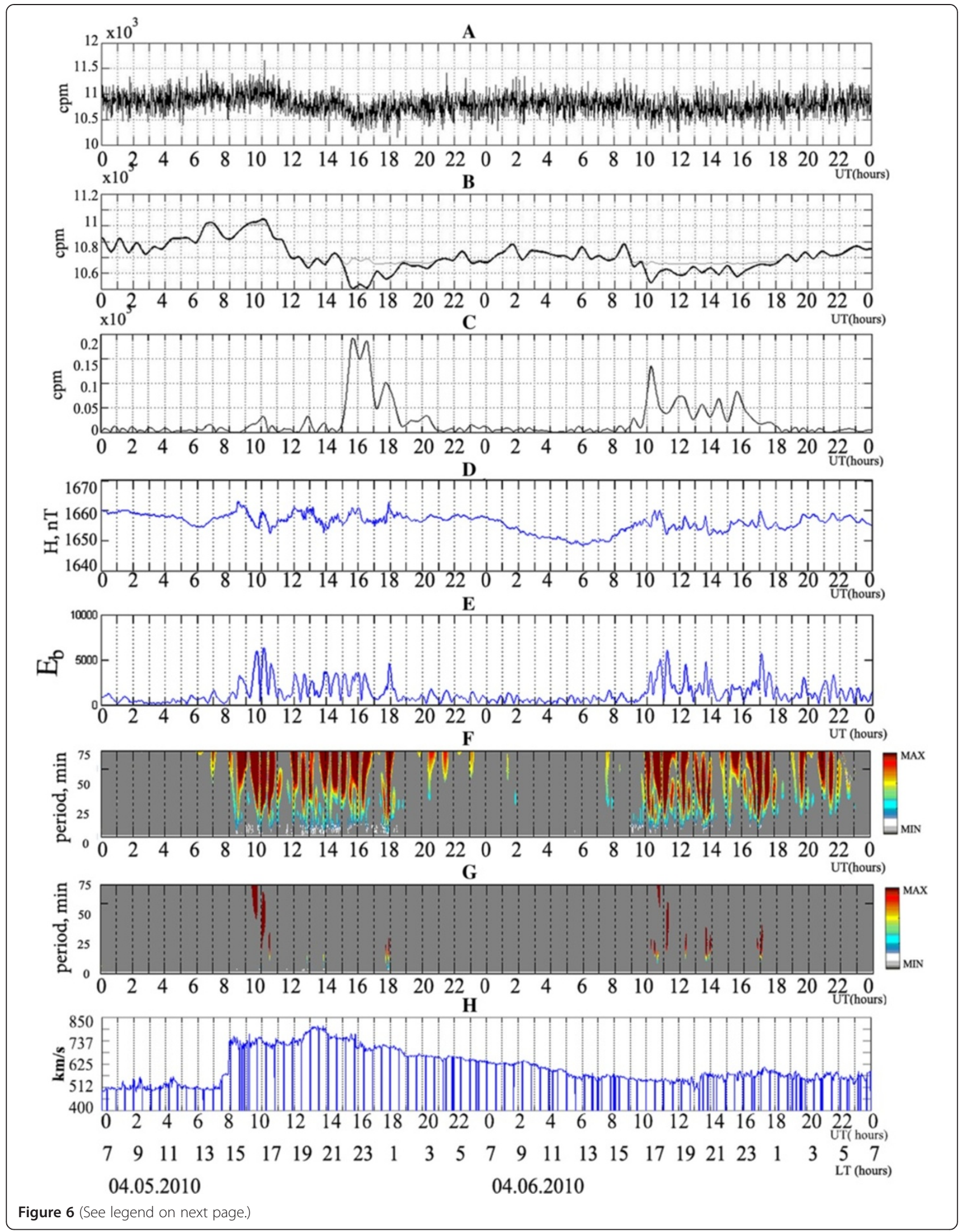


(See figure on previous page.)

Figure 6 Results of processing of the data of geomagnetic field and cosmic rays for the period 5 to 7 April 2010. Novosibirsk station: (A) cosmic ray time series, (B) smoothed component of cosmic ray time series (black) and its approximation by neuron network, (C) neuron network error, (D) H-component of the magnetic field of the Earth at Novosibirsk station (Russia), (E) assessment of geomagnetic disturbance intensity, (F) allocation of the periods of weak and strong geomagnetic disturbances, (G) allocation of the periods of strong geomagnetic disturbances, and $\mathbf{( H )}$ solar wind speed.

increased of geomagnetic activity during a magnetic storm occurring on 24 to 25 June 2009. We estimated thresholds $T_{a, 1}$ and $T_{a, 2}$ by minimizing a posteriori risk, in accordance with rule of decision-making introduced in 'Description of geomagnetic field variations on the basis of wavelets' subsection. K-index was the criterion of estimation of the state of coefficients, where the data used was from stations Paratunka, Yakutsk, and Novosibirsk. Analysis of Figure 3 shows the complex multiscale nature of the process. The results of processing of the data allow us to allocate its general features. Prior to the beginning of the storm, from about 09:00 to 10:40 UT and 21:30 to 23:30 UT on 23 June and from 02:00 to 03:30 UT on 24 June, local increases in the intensity of geomagnetic disturbances were observed at all analyzed stations, and periods of weak geomagnetic activity had been formed. Simultaneous occurrence of disturbances at different stations eliminates the influence of the noise factor and confirms their solar nature. The strongest field perturbations observed from 4:57 to 06:43 UT coincide with the moments of the main phase of the storm.

Method of approximation of the time variation of cosmic rays and allocation of anomalous changes in its dynamic on the basis of combination of multiresolution wavelet decompositions and neural networks

We consider the time variation of cosmic rays as $f_{j}(t)$. Let us assume that the initial data space is $V_{0}=\operatorname{clos}_{L^{2}(R)}$ $\left(\phi\left(2^{0} t-n\right)\right)$ (i.e., $\left.j=0\right)$.

By performing multiresolution wavelet decomposition of the function $f_{0}(t)$ to the level $m$, we obtain its representation in the following form (see Equation 5):

$$
\begin{aligned}
f_{0}(t) & =g_{-1}(t)+g_{-2}(t)+\ldots+g_{-m}(t)+f_{-m}(t) \\
& =\sum_{j=-1}^{-m} \sum_{n} d_{j, n} \Psi_{j, n}(t)+\sum_{n} c_{-m, n} \phi_{-m, n}(t) .
\end{aligned}
$$

For the smooth component $f_{-m}(t)=\sum_{n} c_{-m, n} \phi_{-m . n}(t)$, we perform the operation of wavelet reconstruction:

$$
f_{0}^{(-m)}(t)=\sum_{n} c_{0, n}^{(-m)} \phi_{0, n}(t)
$$

where the upper index $(-m)$ corresponds to the resolution of the component before the operation of the wavelet reconstruction.
Further, on the basis of the neural network for reconstructed components, we constructed the following mapping:

$$
y: f_{0}^{(-m)} \rightarrow f_{0}^{*(-m)}
$$

To construct the mapping, we used a feedforward neural network with variable structure, which is a network in which the structure is determined by minimizing the error on the training set. The training set is formed from the data registered during quiet periods. In this case, the trained neural network reproduced regular variations of the data being approximated, which is typical for quiet conditions. Network training was performed on the basis of the back error propagation algorithm (Haykin 1999).

If in constructed mapping, $\hat{f}_{0}^{*(-m)}$ is the actual output of the network and $f_{0}^{*(-m)}$ is desired, then $f_{0}^{*(-m)}=y\left(f_{0}^{(-m)}\right)$ is an unknown function, and $\hat{f}_{0}^{*(-m)}$ is its approximation, which is reproduced by the neural network. The advantage of the neural network representation of the approximated function is greater flexibility of the basic functions and their ability to adapt (Haykin 1999). When the input of the trained network is the function values from the interval $\left[t_{n}-Q+1, t_{n}\right]$, the network is able to calculate the preemption of its value in the time interval $\left[t_{n}+1, t_{n}+I\right]$, where $t_{n}$ is the current discrete time, and $I$ is the length of the preemption interval.

Network error (approximation error) at time moment $t_{n}$ is determined as the difference between the desired $f_{0}{ }^{*}(-m)$ and actual $\hat{f}_{0}^{*(-m)}$ output values of the function:

$$
e_{m}\left[t_{n}\right]=\sum_{i=1}^{I}\left|f_{0, i}^{*(-m)}\left[t_{n}\right]-\hat{f}_{0, i}^{*(-m)}\left[t_{n}\right]\right|
$$

where $i$ is the step of the feedforward network of the data, and square brackets denote the discrete time.

The algorithm for constructing the network and choosing the level of the wavelet decomposition, based on minimization of the approximation error, is listed below.

If anomalous variations occur in the time variations of the data, the absolute value of the network error would increase. Therefore, the allocation of anomalous changes 


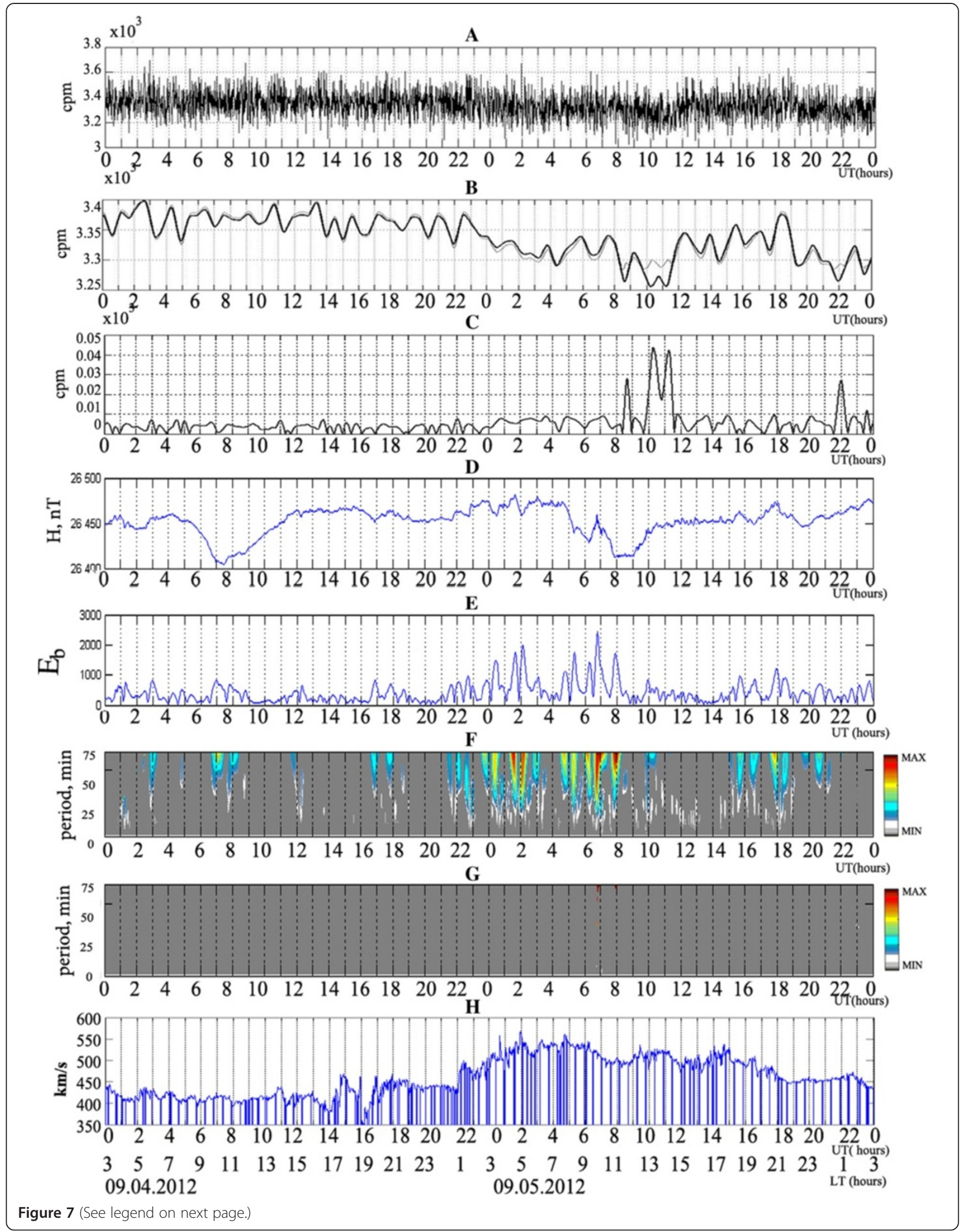


(See figure on previous page.)

Figure 7 Results of processing of the data of geomagnetic field and cosmic rays for the period 4 to 6 September 2012. Athens station: (A) cosmic ray time series, (B) smoothed component of cosmic ray time series (black) and its approximation by neuron network, (C) neuron network error, (D) H-component of the magnetic field of the Earth at Nea-Makri station (Greece), (E) assessment of geomagnetic disturbance intensity, (F) allocation of the periods of weak and strong geomagnetic disturbances, (G) allocation of the periods of strong geomagnetic disturbances, and $\mathbf{( H )}$ solar wind speed.

in the dynamics of the time series could be based, for example, on verifying the condition:

$$
E_{m, U}=\frac{1}{U} \sum_{n=1}^{U} e_{m}\left[t_{n}\right]>T
$$

where $U$ is the length of the observation window, and $T$ is a preassigned threshold.

\section{Algorithm for constructing a neural network and choosing the level wavelet decomposition}

Step 1. We obtain representation of a smoothed component of the series in the form $f_{0}^{(-m)}(t)=\sum_{n} c_{0, n}^{(-m)} \phi_{0, n}(t)$, where $m=1$.

Step 2. Data array $\left\{c_{0, n}^{(-m)}\right\}_{n=1}^{N}$, where $N$ is the array length and is divided into blocks of $\left\{c_{0, n}^{(-m)}\right\}_{n=1}^{Q},\left\{c_{0, n}^{(-m)}\right\}_{n=2}^{Q+1}, \ldots$, $\left\{c_{0, n}^{(-m)}\right\}_{n=N-Q+1}^{N}$. The block length is $Q=6$.

Step 3. From these data blocks, we form a training matrix with the dimensions of $Q \times V$, where $Q$ is the length of the input vector of the network and $V$ is the number of training vectors.

Step 4. We build a network with a variable structure.

Step 5. Using the test data $\left\{c_{0, l}^{(-m)}\right\}_{l=l_{0}}^{L}$, we estimate a network error:

$$
E_{m}=\sum_{l=1}^{L} \sum_{i=1}^{I}\left|\varepsilon_{i}^{(-m)}[l]\right|
$$

where $\varepsilon_{i}^{(-m)}[l]=\hat{c}_{0, l}^{i,(-m)}-c_{0, l}^{i,(-m)}$ is network error at a discrete moment of time $l$ in $i$ steps of preemption, where $c_{0, l}^{i,(-m)}$ is desired; $\hat{c}_{0, l}^{i,(-m)}$ is the actual output value of the network at a discrete moment of time $l$ in $i$ steps of preemption; $I$ is the length of the output vector network; and $L$ is the length of the array of test data.

Step 6. We estimate the difference: $\Delta=E_{m}-E_{m-1}$. If $\Delta<=0$, then go to step 7. If $\Delta>0$, then the required level of decomposition is $m^{*}=m-1$, and we do not perform step 7 .

Step 7. If $m \leq \log _{2} N$, where the maximum allowable level of decomposition $M$ is determined by the length of the array data $N: M \leq \log _{2} N$, we increase by 1 level of the decomposition $(m=m+1)$ and perform steps 2 to 5. If $m>\log _{2} N$, then $m^{*}=m$ is the desired level of decomposition.

\section{Choice of wavelet}

In the present study, a multiresolution wavelet decomposition of the function $f_{0}(t)$ (Equation (18)) was performed using a wavelet from the coiflets family. Coiflets are orthogonal functions that support the smallest size with a sufficient number of zero moments in the scaling function $\phi$ (Daubechies 1992):

$$
\int_{-\infty}^{+\infty} t^{r} \phi(t) d t=0 \text { with } r=\overline{1, p} .
$$

This property provides the best approximation of the smoothed components of multiresolution wavelet decompositions (Daubechies 1992). If a function $f$ belongs to $C^{r}$, which is the space of $r$ times of continuously differentiable functions, in the neighborhood of $2^{-m} n$ with $r \leq p$, then:

$$
2^{-m / 2}\left\langle f, \phi_{-m, n}\right\rangle \approx f\left(2^{-m} n\right)+O\left(2^{-m(r+1)}\right) .
$$

However, the order of approximation increases with the growth of $p$, resulting in coiflets that support size $3 p-1$.

Approximation of the data of cosmic rays with the least error was obtained by using coiflets with $p=3$.

Based on the proposed method, we constructed neural networks for stations Novosibirsk, Athens, Apatity, and Cape Schmidt, which approximate time variations of the smoothed component of cosmic rays and uses the neutron monitor data. To train the networks, we used minute data for the period from 2005 to 2013 years. Cosmic ray variations are influenced by electromagnetic conditions in the solar system; therefore, we used geomagnetic field data as an indicator of anomalous behavior of these conditions. Thus, the training set of neural networks was formed from data registered during periods of quiet geomagnetic field (Akasofu and Chapman 1972; Bishop 1995; Rybàk et al. 2001).

To consider the changes in the level of solar activity, the training of neural networks is performed separately for each year (Kóta and Somogyi 1969; Kudela et al. 1999; Rybàk et al. 2001). The level of the model time series depends on the training sample. The increase in the absolute value of the error of the trained neural network indicates a change in the internal structure of the 


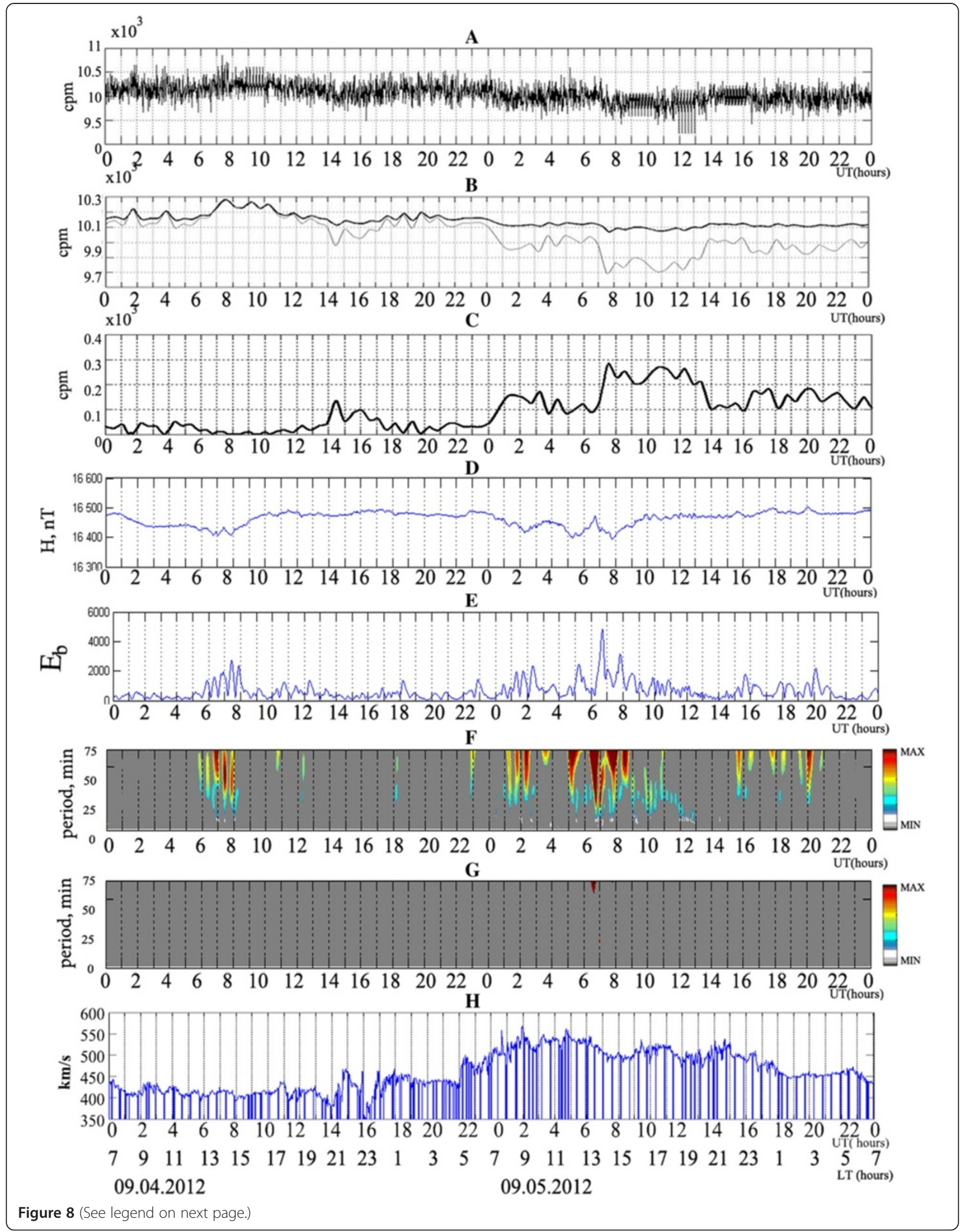


(See figure on previous page.)

Figure 8 Results of processing of the data of geomagnetic field and cosmic rays for the period 4 to 6 September 2012 . Novosibirsk station: (A) cosmic ray time series, (B) smoothed component of cosmic ray time series (black) and its approximation by neuron network, (C) neuron network error, (D) H-component of the magnetic field of the Earth at Novosibirsk station (Russia), (E) assessment of geomagnetic disturbance intensity, (F) allocation of the periods of weak and strong geomagnetic disturbances, (G) allocation of the periods of strong geomagnetic disturbances, and $\mathbf{( H )}$ solar wind speed.

time series and the occurrence of anomalies in cosmic rays.

The constructed neural networks have a three-layer structure and perform the following data conversion:

$$
c_{j, n+1}(t)=\alpha_{X}^{3}\left(\sum_{\varphi} \omega_{\chi \varphi}^{3} \alpha_{\varphi}^{2}\left(\sum_{\beta} \omega_{\varphi \beta}^{2} \alpha_{\beta}^{1}\left(\sum_{n} \omega_{\beta n}^{1} c_{j, n}(t)\right)\right)\right),
$$

where $\omega_{\beta n}^{1}$ is the weight of neuron $\beta$ of the input layer of the network, $\omega_{\varphi \beta}^{2}$ is the weight of the neuron $\varphi$ of the hidden layer of the network, $\omega_{X \varphi}^{3}$ is the weight of a neuron $X$ of the output layer of the network, and $\alpha_{\beta}^{1}(z)=\alpha_{\varphi}^{2}(z)=\frac{2}{(1+\exp (-2 z))}-1, \alpha_{\chi}^{3}(z)=a \times z+b, j=-5$.

Figure 4 shows the results of neural networks constructed for Novosibirsk and Athens stations for a period of strong magnetic storms recorded 1 to 31 March 2012. The first storm occurred on 7 to 10 March. The Forbush effect was associated with this storm, which emerged on $8 \mathrm{March}$, and was expressed in a strong decrease in the level of cosmic rays (up to 10\%) at both stations. During the Forbush effect, the absolute values of the network errors significantly increase (up to 10 times), which allowed us to allocate those time periods. A comparison of the geomagnetic data shows that the strongest geomagnetic disturbances were observed during periods of anomalous behavior of cosmic ray dynamics. During the second magnetic storm (15 to $17 \mathrm{March}$ ), the moments of strong increases in the intensity of geomagnetic disturbances coincided with the local lowering of the level of cosmic rays and had a more clear manifestation at the Novosibirsk station. Restoration of the level of cosmic rays occurred after the end of the storm.

\section{Experimental results}

Figures 5 and 6 show the results of the processing of the geomagnetic field and the data of cosmic rays during magnetic storms that occurred 5 to 7 April 2010. Figures 7 and 8 show the same results but for magnetic storms that occurred 5 to 6 September 2012 .

The first analyzed magnetic storm was registered on Earth on 5 April 2010, at 08.26 UT, as the sudden commencement (SC) of the magnetic storm. The speed of the solar wind had increased to 750 to $900 \mathrm{~km} / \mathrm{s}$. A globally observed intense substorm emerged $30 \mathrm{~min}$ later in the Earth's magnetosphere (Kleimenova et al. 2013).

Analysis of Figures 5 and 6 reveals that a local decrease of the level of cosmic rays occurred simultaneously with an increase of geomagnetic disturbance intensity. The error of neural networks increased fourfold at the Athens station and 2.5 times at the Novosibirsk station in comparison with the quiet period. Decreases in the level of cosmic rays at both stations were accompanied by the most severe disturbances of the geomagnetic field. However, variations of cosmic rays had different behaviors. At the Athens station, a short Forbush effect with rapid recovery peaked at 20:00 UT, in which the neural network error increased in threefold in comparison with the quiet period. At the Novosibirsk station, the Forbush effect was longer and peaked at 16:00 UT; the neural network error increased tenfold in comparison with the quiet period. The intensity of the geomagnetic field disturbances reached maximum values during 9:26 to 10:07 UT.

The second analyzed magnetic storm was recorded on Earth on 5 September 2012. The polar wind velocity reached values of 500 to $600 \mathrm{~km} / \mathrm{s}$ due to the arrival of the accelerated flow from the coronal mass ejection (CME) that occurred on 2 September 2012. Detailed analysis of Figures 7 and 8 reveals that local increases in intensity of geomagnetic disturbances emerged at the analyzed stations in different frequency ranges and that periods of weak geomagnetic activity had been formed on the eve of storm (approximately 06:00 to 08:00 UT, $10: 30$ to $12: 30 \mathrm{UT}$, and 17:00 to 18: $00 \mathrm{UT}$ on 4 September and from 22:00 UT until the start of the storm). The arrival of the shock wave occurred at 1:00 UT on 5 September, and both stations simultaneously recorded the beginning of the Forbush effect; an increase in the error of the neural network was recorded at the Novosibirsk station. Maximal values of intensity field disturbances were recorded on 5 September during 6:43 to 6:48 UT, which coincides with the periods of strong geomagnetic activity. At the Novosibirsk station, the maximum of the lowering of the level of cosmic rays and neural network error had increased in ten times in comparison with the quiet period. It should be noted that the Forbush effect registered at the Athens station was short, and the cosmic ray level recovered fast, whereas the Forbush effect registered at Novosibirsk station was more extended. 


\section{Conclusions}

We proposed a model of geomagnetic field variations that allows us to describe the tranquil dynamics and disturbances occurring in periods of increased geomagnetic activity. On the basis of this model, we developed methods for assessing changes in energy characteristics of the field and allocation of periods of weak and strong geomagnetic activity. The analysis showed that in periods of increased geomagnetic activity, the developed tools allowed us to record the onset of geomagnetic disturbances and to receive quantitative estimates of the power of the geomagnetic field disturbance. Such applications allowed us to perform detailed analysis of geomagnetic field variations for different registration stations during strong magnetic storms.

A slight increase in geomagnetic activity, which is associated with the approaching storm, was observed in different frequency ranges at local moments on the eves of storms.

Moreover, we proposed methods of approximation of the time variation of cosmic ray data and allocation of anomalous changes occurring during periods of high solar activity. The methods for Novosibirsk, Athens, Cape Schmidt, and Apatity stations were built in neural networks that approximate the time variation in the smoothed component of the cosmic rays. In the training of the networks, we used data recorded in 2005 to 2013. This method allowed us to study the structure of the data of cosmic rays in detail and to allocate abnormal changes in their dynamics during periods of the Forbush effects.

Joint analysis of the data of the geomagnetic field and cosmic rays in the periods of strong magnetic storms showed that the strongest geomagnetic field perturbations were observed in periods of anomalous changes in the dynamics of cosmic rays and could be registered on the basis of the proposed applications.

Future plans include adaptation of the developed tools for a larger number of registration stations and creation of an integrated software environment that would provide the possibility of spatio-temporal data analysis in an online mode.

\section{Competing interests}

The authors declare that they have no competing interests.

\section{Authors' contributions}

OVM proposed a method for describing variations of the geomagnetic field based on wavelets and an approach to solving the problem of data approximation of the cosmic rays based on a combination of multiresolution wavelet decompositions with neural networks. ISS proposed the computing solutions for estimating changes in the energy characteristics of the field and the allocation of periods of increased geomagnetic activity in addition to algorithms for processing and analysis of geomagnetic data. TLZ developed a method for approximating the time variation of cosmic rays and allocation of abnormal changes in their dynamics based on multiresolution wavelet decompositions and neural networks in addition to algorithms for processing and analysis of the data of cosmic rays. All authors read and approved the final manuscript.

\section{Acknowledgements}

This research is supported by a grant of Russian Academic Fund No. 14-11-00194 We thank researchers of the NMDB project (www.nmdb.eu/) for providing highresolution neutron monitor data and those at Intermagnet (www.intermagnet.org) for promoting high standards of magnetic observatory practices.

\section{Author details}

${ }^{1}$ Institute of Cosmophysical Researches and Radio Wave Propagation, Mirnaya Str., 7, Kamchatka Region, Elizovskiy District, Paratunka 684034, Russia. ${ }^{2}$ Kamchatka State Technical University, Klyuchevskaya Street 35, 683003 Petropavlovsk-Kamchatsky, Russia. ${ }^{3}$ Saint Petersburg Electrotechnical University "LETI", Ulitsa Professora Popova, 5, St Petersburg, Russia.

Received: 31 March 2014 Accepted: 29 October 2014

Published online: 21 November 2014

\section{References}

Akasofu SI, Chapman S (1972) Solar-terrestrial physics. Oxford University Press, Oxford Bartels J, Heck NH, Johnston HF (1939) The three hour range index measuring geomagnetic activity. J Geophys Res 44:411-454

Bishop CM (1995) Neural networks for pattern recognition. Oxford University Press Inc., New York

Chui CK (1992) An introduction in wavelets. Academic Press, New York

Daubechies I (1992) Ten lectures on wavelets. CBMS-NSF lecture notes nr. 61. SIAM, Philadelphia

Hafez AG, Ghamry E, Yayama H, Yumoto K (2013) Systematic examination of the geomagnetic storm sudden commencement using multi resolution analysis. Adv Space Res 51:39-49

Haykin S (1999) Neural networks: a comprehensive foundation, 2nd edn. Prentice-Hall, New York

Ivanov W, Rotanova NM, Kovalevskaya EV (2001) The wavelet analysis as applied to the study of geomagnetic disturbances. Geomagn Aeron 41:583-591

Jach A, Kokoszka P, Sojka J, Zhu L (2006) Wavelet-based index of magnetic storm activity. J Geophys Res 111:A09215, doi:10.1029/2006JA011635

Kleimenova NG, Zelinskii NR, Kozyreva OV, Malysheva LM, Solov'ev AA, Bogoutdinov SR (2013) PC3 geomagnetic pulsations at near-equatorial latitudes at the initial phase of the magnetic storm of April 5, 2010. Geomagn Aeron 53:313-320

Kóta J, Somogyi A (1969) Some problems of investigating periodicities of cosmic rays. Acta Physica Acad Sci Hung 27:523-548

Kozlov VI, Markov W (2007) Wavelet image of a heliospheric storm in cosmic rays. Geomagn Aeron 47:52-61

Kudela K, Yasue S, Munakata K, Bobik P (1999) Cosmic ray variability at different scales: a wavelet approach. Proceedings of the 26th International Cosmic Ray Conference, Salt Lake City, USA

Levin BR (1963) Theoretical basis of statistical radio techniques. Fizmatgiz, Moscow

Lundstedt H, Liszka L, Lundin R (2005) Solar activity explored with new wavelet methods. Ann Geophys 23:1505-1511, doi:10.5194/angeo-23-1505-2005

Macpherson KP, Conway AJ, Brown JC (2001) Prediction of solar and geomagnetic activity data using neural networks. J Geophys Res 100:735-744

Mallat S (1999) A wavelet tour of signal processing. Academic Press, London

Mandrikova OV, Solovev IS (2013) The method of the extracting disturbance and estimates of the Earth's magnetic field is based on the wavelet-packet. Proceedings of 11th International Conference on Pattern Recognition and Image Analysis, Samara, Russia

Mandrikova OV, Solovjev I, Geppenerc V, Taha A-KR, Klionskiy D (2013) Analysis of the Earth's magnetic field variations on the basis of a wavelet-based approach. Digit Signal Process 23:329-339

Nayar SRP, Radhika VN, Seena PT (2006) Investigation of substorms during geomagnetic storms using wavelet techniques. Proceedings of the ILWS Workshop Goa, India

Paschalis P, Sarlanis C, Mavromichalaki H (2013) Artificial neural network approach of cosmic ray primary data processing. Sol Phys 182(1):303-318

Rotanova N, Bondar T, Ivanov V (2004) Wavelet analysis of secular geomagnetic variations. Geomagn Aeron 44:252-258

Rybàk J, Antalovà A, Storini M (2001) The wavelet analysis of the solar and cosmic-ray data. Space Sci Rev 97:359-362

Segarra A, Curto JJ (2013) Automatic detection of sudden commencements using neural networks. Earth Planets Space 65(7):791-797 
Vecchio A, Laurenza M, Storini M, Carbone V (2012) New insights on cosmic ray modulation through a joint use of nonstationary data-processing methods. Adv Astronomy 2012. doi:10.1155/2012/834247

Woolley JW, Agarwarl PK, Baker J (2010) Modeling and prediction of chaotic systems with artificial neural networks. Int J Numer Methods Fluids 63, doi:10.1002/fld.2117

Xu Z, Zhu L, Sojka J, Kokoszka P, Jach A (2008) An assessment study of the wavelet-based index of magnetic storm activity (WISA) and its comparison to the Dst index. J Atmos Solar Terr Phys 70:1579-1588

Zaourar N, Hamoudi M, Mandea M, Balasis G, Holschneider M (2013) Waveletbased multiscale analysis of geomagnetic disturbance. Earth Planets Space 65(12):1525-1540

doi:10.1186/s40623-014-0148-0

Cite this article as: Mandrikova et al:: Methods of analysis of

geomagnetic field variations and cosmic ray data. Earth, Planets and

Space 2014 66:148.

\section{Submit your manuscript to a SpringerOpen ${ }^{\circ}$ journal and benefit from:}

- Convenient online submission

- Rigorous peer review

- Immediate publication on acceptance

- Open access: articles freely available online

- High visibility within the field

- Retaining the copyright to your article

Submit your next manuscript at $>$ springeropen.com 